\title{
Thermally Driven Steady MHD Mixed Convective Chemically Reacting Flow over a Hot Porous Linearly Stretching Sheet
}

\author{
Okuyade Ighoroje W.A. \\ Department of Mathematics - Statistics, University of Port Harcourt, Port Harcourt, Nigeria \\ wiaokuyade@gmail.com
}

\begin{abstract}
Thermally driven steady mixed convective chemically reacting flow of an electrically conducting viscous incompressible fluid over a linearly stretching sheet in the presence of suction/injection, heat generation/absorption and thermal radiation is investigated. The governing nonlinear equations are transformed into ordinary differential equations using the similarity transformation, and linearized using the perturbation series expansions. The resulting linear similarity equations are solved semi-analytically using the Mathematica 9.0 software. Solutions of the concentration, temperature, velocity, Nusselt number, Sherwood number and skin friction are obtained, and presented graphically to illustrate the effects of the various parameters on the dependent flow variables. The results are extensively discussed. Furthermore, the results obtained are bench-marked with some of those obtained in the earlier studies in literature, and are found to be in consonance.
\end{abstract}

Keywords: $M H D$, mixed convection, suction/injection, stretching sheet, thermal radiation.

MSC No: $76 R_{10}$ and $80 A_{20}$

\section{Introduction}

Convective flow over moving stretching sheets/surfaces with uniform or non-uniform velocity in an ambient fluid has applications in several manufacturing processes. It is relevant in the plastic sheet extrusion or polymer processing, cooling of infinite metallic plate in a cooling bathe, glass blowing, wire and fibre coating, extrusion of molten metal from non-metallic inclusions, and so on.

A manufactured product in the form of a thermal forming stretching sheet at high temperature needs cooling in a fluid system. The properties of the final product depend largely on the rate of cooling, which can be achieved by drawing the sheet in an ambient fluid, thus allowing the fluid to flow over it. Introducing a hot stretching sheet into an ambient flowing fluid may give rise to new situations in the flow field. For example, the heat from the hot sheet is emitted, absorbed into the fluid and transported by convection; fluid can be sucked into or injected out of the stretching sheet, thus making the flow suction/injection induced; the sheet may contain chemicals which may diffuse into the fluid. With the chemicals, the fluid may become electrically conducting, and thus magnetically susceptible. Drawing the sheet in the fluid can be achieved, using applied forces in conjunction with the free convection force, making the problem mixed convective.

For its practical applications, great research interest has been attracted to this domain of study. Some considered the linearly stretching sheet; some the non-linearly stretching sheet (see Motsa and Sibanda [1]); some the exponentially stretching sheet (see Ferdows et al. [2]), and others, the rotating stretching sheet (see Wahiduzzana et al. [3]). Specifically, a number of 
reports exist in literature on the problem of flow over linearly stretching sheets. For example, Nadeem et al. [4] examined the two dimensional flow of Williamson fluid over a stretching sheet using similarity transformations, boundary layer approach and homotopy analysis method, and observed that the velocity and skin friction decrease with the increase in the Williamson parameter. Nadeem et al. [5] studied the steady flow of Casson fluid over a linearly stretching sheet in the presence of a nano-particle using similarity transformation solution and numerical approach, and observed that the Brownian and thermo-phoresic parameters reduce the Nusselt number but increase the Sherwood number. Awad et al. [6] considered the unsteady incompressible flow of a polymer nano composite represented by an Oldroyd-B nano-fluid along a stretching sheet using the spectral relaxation method, and found that the visco-elastic profiles of the fluid resist the motion; the effect of the Brownian motion on the rate of the heat transfer is negligible.

Convective flows under the influence of a magnetic field and chemical reaction occur in many transport processes, both in natural and artificial worlds. Therefore, they are applicable in science and engineering. The phenomena are relevant in the chemical industry, power and cooling industry for drying, chemical vapour deposition on surfaces, cooling of nuclear reactors and petroleum industries. Sakiadis [7] developed a numerical solution using a similarity transformation for steady heat flow over a moving continuous surface. Elbashabeshy and Bazidn [8] studied the flow and heat transfer in a porous medium over a stretching surface with internal heat generation and suction or blowing, and found that the heat transfer in the flow is analyzed only when the surface is held at constant temperature. Arial et al. [9] investigated a visco-elastic non-Newtonian fluid flow past a linearly stretching sheet with partial slip effects; Kandasamy et al. [10] presented a group analysis for the Soret and Dufour effects on free convective heat and mass transfer with thermo-phoresis and chemical reaction over a porous stretching surface in the presence of heat source/sink.

The study has also been extended to include the effects of magnetic field. Magnetohydrodynamic convective heat and mass transfer in porous and non-porous media is of considerable interest in technical field due to its applications in industries, geothermal, high temperature plasma, liquid metal and MHD power generating systems. $\mathrm{Xu}$ [11] considered the convective MHD heat transfer flow over a linearly stretching surface with uniform free stream, and obtained the heat transfer effect. Cortell [12] studied the flow and heat transfer of a fluid through a porous medium over a stretching surface with internal heat generation/absorption and suction/blowing, and obtained analytic solutions for both when the surface temperature is constant and prescribed. They observed that the skin friction is independent of both Prandtl number and source/sink parameter; the increase in the permeability decreases the velocity but increases the temperature; suction decreases the velocity whereas injection increases it. Seddeek and Almushigeh [13] investigated the effects of radiation and variable viscosity on MHD free convective flow and mass transfer over a stretching sheet with chemical reaction. Oahimire and Olajuwon [14] studied the hydrodynamic flow of a viscous fluid near a stagnation point on a linearly stretching sheet with variable thermal conductivity and heat source/sink analytically using similarity transformation and perturbation technique. They observed among others, that the increase in heat generation decreases the temperature profiles, and the increase in magnetic field increases the temperature profiles showing that the temperature of the fluid near the sheet is higher. Shateyi [15] considered the flow of a Maxwell fluid past a vertically stretching sheet in a Darcian porous medium under the influence of thermo-phoresis, thermal radiation and uniform chemical reaction using the method of similarity and Chesbyshev pseudo-spectral collocation method. He saw that the temperature increases with the increase in magnetic field, porosity, Deborah number and thermal radiation parameter but decreases with the increase in Prandtl number.

Many processes in engineering occur at higher temperature, and the quality of the final product depends, to a great extent, on the rate of cooling and heat controlling factors. Therefore, the knowledge of radiative heat transfer has become very important in the design of pertinent equipment. Radiative heat and mass transfer play important roles in the design of fins, steel 
rolling, nuclear power plants, gas turbines, and various propulsion devices for aircraft, missiles, satellites, and space vehicles. Upon these imports, Vyas and Srivastava [16] investigated the radiation effects on the MHD flow over a non-isothermal stretching sheet in a porous medium. Motsa and Shateyi [17] studied the problem of unsteady heat and mass transfer from a stretching surface embedded in a porous medium with suction/injection and thermal radiation effects using successive linearization method, and showed that the results obtained are more accurate and efficient than those obtained by the homotopy method and Runge-Kutta numerical scheme. Vyas and Srivastava [18] studied the radiation effects on MHD boundary layer flow in a porous medium over a non-isothermal stretching sheet in presence of dissipation. Ibrahim [19] studied the steady two-dimensional radiative MHD boundary layer flow of an incompressible, viscous, electrically conducting fluid caused by a non-isothermal linearly stretching sheet placed at the bottom of fluid saturated porous medium in the presence of viscous dissipation and chemical reaction using the similarity transformations and numerical shooting method. They observed that the skin friction, Nusselt number, and Sherwood number decrease with the increase of the magnetic field parameter or permeability parameter;

the velocity profiles increase with the increase of Grashof number or modified Grashof number. Moreover, they noticed that both velocity and the temperature profiles decrease with the increase of the wall temperature parameter; the increase of the thermal radiation parameter decreases the velocity and temperature profiles; the Schmidt number decreases the concentration field. Hayat et al. [20] studied the mixed convective MHD flow of a non-Newtonian nano-fluid over a linearly stretching sheet in the presence of thermal radiation, heat source/sink and first order chemical reaction using the similarity transformation and series solutions. They noticed amongst others, that the increase in momentum boundary layer thickness, velocity profiles and thermophoretic parameters increase the temperature and concentration profiles; the rise in the Brownian motion parameter increases the skin friction coefficient and Sherwood number but decreases the local Nusselt number. Sandeep et al. [21] considered the boundary layer flow of a thermophoretic MHD dissipative nano-fluid over an unsteady stretching sheet in a porous medium with space and time dependent heat source/sink using similarity transformation and numerical shooting technique. They noticed amongst others, that the magnetic field and porosity parameters reduce the velocity but increase the temperature profiles; thermo-phoresis, Brownian motion parameters and Lewis number do not influence the velocity. Sreedevi et al. [22] examined the influence of the radiation absorption variable viscosity and hall current of a MHD free convective heat and mass transfer flow over a linearly stretching sheet in the presence of heat generation/absorption using the method of similarity transformation and numerical finite difference scheme. They observed that an increase in the radiation absorption parameter increases the velocity, temperature, concentration, Nusselt and Sherwood numbers. Adeniyan [23] investigated the steady two-dimensional hydro-magnetic free convective boundary layer flow over a linearly stretching vertical porous sheet embedded in an expanse of incompressible, electrically conducting fluid in the presence of combined influence of stress work, exponentially decaying heat generation, Soret and Dufour numbers, and suction/injection parameter using a numerical approach. He observed, amongst others, that the field velocity increases in the boundary layer with the increase in the buoyancy parameters, Eckert number, Schmidt number, thermal conductivity variation, heat generation and chemical reaction.

Similarly, the flow involving chemical reaction has been well investigated. Takhar et al. [24] examined the flow and mass transfer characteristics of a viscous electrically conducting fluid over a continuous stretching surface with chemically reacting species and non-zero slot velocity using an implicit finite difference scheme. They noticed that the magnetic field increases the surface skin friction but reduces the surface mass transfer; the surface mass transfer depends on the Schmidt number and reaction rate, which increases as they increase. Makinde and Sibanda [25] investigated the effects of first-order homogeneous chemical reaction on a twodimensional boundary layer flow past a stretching surface in the presence of internal heat generation using the numerical shooting technique and sixth-order Runge-Kutta scheme. They found that for positive values of buoyancy parameter the local skin friction and mass transfer 
coefficient increase with the increase in Eckert number and Schmidt number while the heat transfer coefficient decreases with the increase in Eckert number and Schmidt number; both velocity and temperature profiles increase when the heat generation parameter increases.

Takhar et al. [24] investigated the flow and mass transfer characteristics of a viscous electrically conducting fluid on a continuous stretching surface with chemically reacting species using an implicit finite difference scheme. They did not consider the effects of thermal differentials. Therefore, this paper examines the roles of thermal differentials and other chosen parameters in the motion of linearly stretching sheet in a fluid.

This paper is organized in the following manner: section 2 is the methodology; sections 3 and 4 hold the results and discussion respectively, and section 5 is the conclusion.

\section{The Physics of Problem and Mathematical Formulation}

We consider the problem of steady MHD two-dimensional flow of fluid over an accelerating semi-infinite linearly stretching surface. We assume that two equal and opposite forces are applied along the $\mathrm{x}$-axis so that the wall is stretched keeping the origin fixed, thus giving the sheet a unidirectional orientation; that these equal and opposite forces partly generate the flow. The $\mathrm{x}$-axis is taken to be the direction along the porous surface, and this coincides with $\mathrm{y}=0$.More so, we assume the fluid is incompressible and Newtonian; the sheet is permeable, and coated with some chemical species whose concentration varies linearly along the length of the surface; suction or injection is imposed at the boundary; a constant external magnetic field is applied normal to the sheet surface; there is a first order chemical reaction; the sheet is maintained at wall temperature and concentration higher the ambient temperature and concentration of the fluid; there are buoyancy forces arising from the concentration and temperature gradients; viscous dissipation is negligible. Therefore, if $\left(u^{\prime}, v^{\prime}, w^{\prime}\right)$ are the velocity vectors with respect to the orthogonal Cartesian coordinate directions $\left(x^{\prime}, y^{\prime}, z^{\prime}\right)$ and are symmetrical about the $z^{\prime}$ axis such that the variations about $z^{\prime}$ is zero, the velocity and coordinate vectors become $\left(u^{\prime}, v^{\prime}\right)$ and $\left(x^{\prime}, y^{\prime}\right)$ respectively; then the mathematical models for the continuity, momentum, energy and diffusion equations, using the Boussinesq approximations are:

$$
\begin{aligned}
& \frac{\partial u^{\prime}}{\partial x^{\prime}}+\frac{\partial v^{\prime}}{\partial y^{\prime}}=0 \\
& u^{\prime} \frac{\partial u^{\prime}}{\partial x^{\prime}}+v^{\prime} \frac{\partial u^{\prime}}{\partial y^{\prime}}=v \frac{\partial^{2} u^{\prime}}{\partial y^{\prime 2}}-\left(\frac{v}{\kappa}+\frac{\sigma_{c} B_{0}^{2}}{\rho \mu \mu_{m}}\right) u^{\prime}-g \beta_{t}\left(T^{\prime}-T_{\infty}\right)-g \beta_{c}\left(C^{\prime}-C_{\infty}\right) \\
& u^{\prime} \frac{\partial u^{\prime}}{\partial x^{\prime}}+v^{\prime} \frac{\partial u^{\prime}}{\partial y^{\prime}}=\frac{k}{\rho C_{p}} \frac{\partial^{2} T^{\prime}}{\partial y^{\prime 2}}+\frac{Q}{\rho C_{p}}\left(T^{\prime}-T_{\infty}\right)-\frac{1}{\rho C_{p}} \frac{\partial q_{y}}{\partial y^{\prime}} \\
& u^{\prime} \frac{\partial C^{\prime}}{\partial x^{\prime}}+v^{\prime} \frac{\partial^{2} C^{\prime}}{\partial y^{\prime}}=D \frac{\partial^{2} C^{\prime}}{\partial y^{\prime 2}}-k_{r}^{2}\left(C^{\prime}-C_{\infty}\right) \\
& \frac{\partial^{2} q_{r}}{\partial y^{\prime 2}}-3 \alpha^{2} q_{y}+4 \sigma \alpha \frac{\partial T^{\prime 4}}{d y^{\prime}}=0 \\
& u^{\prime}=U(x)=a x^{\prime} \\
& v^{\prime}=-V_{w} \\
& T^{\prime}=T_{w}=T_{\infty}+A_{1} x^{\prime} \\
& \text { at } y=0 \\
& C^{\prime}=C_{w}=C_{\infty}+A_{1} x^{\prime}
\end{aligned}
$$




$$
\left.\begin{array}{l}
u^{\prime}\left(x^{\prime}, \infty\right)=0 \\
v^{\prime}(x, \infty)=0 \\
T^{\prime}\left(x^{\prime}, \infty\right)=T_{\infty} \\
C^{\prime}\left(x^{\prime}, \infty\right)=C_{\infty}
\end{array}\right\} \quad \text { at } y^{\prime}=\infty
$$

where $T^{\prime}$ is the temperature of the fluid, $T_{\infty}$ is the ambient temperature of the fluid, $C^{\prime}$ is the concentration of the fluid, $C_{\infty}$ is the ambient concentration of the fluid, $\mathrm{p}$ is the pressure. $\mu$ is the absolute/dynamic viscosity of fluid, $v$ is the kinematic viscosity of the fluid, $\rho$ is the fluid density ,$\sigma_{e}$ is the electrical conductivity of the fluid, $\mathrm{g}$ is acceleration due to gravity. $\kappa$ is the permeability of the sheet, $\beta_{t}$ is the volumetric-expansion coefficient of the fluid due to temperature, $\beta_{c}$ is the volumetric-expansion coefficient of the fluid due to concentration, $B_{o}^{2}$ is the magnetic field flux. $\mathrm{k}$ is the thermal diffusivity, $C_{p}$ is the specific heat capacity at constant pressure, $\mathrm{Q}$ is the volumetric rate of heat generation/absorption, $q_{y}$ is the heat flux at the wall of the plate. $\mathrm{D}$ is the diffusion coefficient, $k_{r}^{2}$ is the chemical reaction rate constant, $U(x)$ is the free stream velocity of the fluid in the x-axis, $V_{w}$ is the suction/injection. $T_{w}$ is the temperature at the wall of the plate surface, $C_{w}$ is the concentration at the wall of the plate surface, $\sigma$ is the Stefan-Boltzman constant, $\alpha$ is the depth of penetration of the rays into the fluid.

Furthermore, we assume the medium is optically thin, that is, its density is relatively low; the medium is transparent, and the depth of penetration of radiant rays (or the mean radiation absorption) into it is such that $\alpha<<1$ (see Vincetti and Krugger [26], Siegel and Howel [27], Alagoa et al. [28], Israel-Cookey and Nwaigwe [29]).To this end, equation (5) reduces to

$$
\frac{\partial^{2} q_{r}}{\partial y^{\prime 2}}=-4 \sigma \alpha \frac{\partial T^{\prime 4}}{d y^{\prime}}
$$

Similarly, we assume the reference temperature is not much different from those of the adjacent layers of the fluid such that $T^{\prime}-T_{\infty}=\theta$, where $\theta$ is a temperature difference between adjacent fluid layers, otherwise called a non-constant small temperature correction parameter, and can be expressed in Taylor series as $T^{4}=4 T_{\infty}^{3} T^{\prime}-3 T_{\infty}^{3}$

Substituting this into equation (8) and integrating, we obtain

$$
\frac{\partial q_{r}}{\partial y^{\prime}}=-16 \sigma \alpha T_{\infty}^{\prime 3} T^{\prime}
$$

where $O\left(3 T_{\infty}^{\prime 3}\right)$ is neglected

By equation (9), equation (3) becomes

$$
u^{\prime} \frac{\partial u^{\prime}}{\partial x^{\prime}}+v^{\prime} \frac{\partial u^{\prime}}{\partial y^{\prime}}=\frac{k}{\rho C_{p}} \frac{\partial^{2} T^{\prime}}{\partial y^{\prime 2}}+\frac{Q}{\rho C_{p}}\left(T^{\prime}-T_{\infty}\right)+\frac{16 \sigma \alpha T_{\infty}^{3} T^{\prime}}{\rho C_{p}}
$$

Introducing the following dimensionless terms:

$$
\begin{gathered}
u=\frac{u^{\prime}}{U}, v=\frac{v^{\prime}}{U}, x=\frac{x^{\prime}}{l}, y=\frac{y^{\prime}}{l}, \Theta=\frac{T^{\prime}-T_{\infty}}{T_{w}-T_{\infty}}, \Phi=\frac{C^{\prime}-C_{\infty}}{C_{w}-C_{\infty}}, \\
\operatorname{Pr}=\frac{\mu C_{p}}{k}, S c=\frac{v}{D}, \Delta=\frac{Q l^{2}}{\rho C_{p}}, \delta_{1}^{2}=\frac{k_{r}^{2} l}{D}, R a=\frac{16 \sigma \alpha T_{\infty}^{3} l^{2}}{k}, \\
N=\frac{\beta_{2}\left(C_{w}-C_{\infty}\right)}{\beta_{1}\left(T_{w}-T_{\infty}\right)}, M^{2}=\frac{\sigma_{c} B_{0}^{2}}{\rho \mu \mu_{m}}, \chi^{2}=\frac{l^{2}}{\kappa}, G r=\frac{g l^{2}}{v U}
\end{gathered}
$$


where $\Theta$ is the dimensionless temperature, $\Phi$ is the dimensionless concentration, $\mathrm{N}$ is the buoyancy ratio, $M^{2}$ is the Hartmann number, $\chi^{2}$ is the Darcy number, $\mathrm{Gr}$ is the Grassh of number, $\operatorname{Pr}$ is the Prandtl number, Sc is the Schmidt number. $\mathrm{U}$ is the free stream velocity of the fluid, $\delta_{1}^{2}$ is the dimensionless chemical reaction rate, $\Delta$ is the dimensionless heat generation generation/absorption parameter) into equations (1), (2), (10) and (4), we get

$$
\begin{aligned}
& \frac{\partial u}{\partial x}+\frac{\partial v}{\partial y}=0 \\
& \frac{\partial^{2} u}{\partial y^{2}}-M_{1}^{2} u=u \frac{\partial u}{\partial x}+v \frac{\partial u}{\partial y}-G r(\Theta+N \Phi) \\
& \frac{1}{\operatorname{Pr}} \frac{\partial^{2} \Theta}{\partial y^{2}}+\lambda \Theta=u \frac{\partial \Theta}{\partial x}+v \frac{\partial \Theta}{\partial y}-G r(\Theta+N \Phi) \\
& \frac{1}{S c} \frac{\partial^{2} \Phi}{\partial y^{2}}-\delta_{1}^{2} \Phi=u \frac{\partial \Phi}{\partial x}+v \frac{\partial \Phi}{\partial y}
\end{aligned}
$$

where $M_{1}^{2}=M^{2}+\chi^{2}, \lambda=\Delta+R a$

with the boundary conditions

$$
\begin{aligned}
& u=1, v=0, \Theta=1, \Phi=1 \quad \text { at } \quad y=0 \\
& u=0, v=0, \Theta=0, \Phi=0 \quad \text { at } \quad y=\infty
\end{aligned}
$$

Furthermore, we invoke the stream function and similarity solutions

$$
\eta=\left(\frac{U}{v x}\right)^{\frac{1}{2}} y, \psi=(U v x)^{\frac{1}{2}} f(\eta), u=\frac{\partial \psi}{\partial y}, v=-\frac{\partial \psi}{\partial x}
$$

(where $\psi$ is the stream function, $f$ is the similarity velocity function, $\eta$ is the similarity independent variable) into equations (12) - (17) to have

$$
\begin{array}{r}
f^{\prime}=0 \\
f^{\prime \prime \prime}+f f^{\prime \prime}-\left(f^{\prime}\right)^{2}-M_{1}^{2} f^{\prime}+G r(\Theta+N \Phi)=0 \\
\frac{1}{\operatorname{Pr}} \Theta^{\prime \prime}+f \Theta '-\Theta f^{\prime}+\lambda \Theta=0 \\
\frac{1}{S c} \Phi^{\prime \prime}+f \Phi^{\prime}-\Phi f^{\prime}-\delta_{1}^{2} \Phi=0
\end{array}
$$

(where the primes denote differentiation with respect to $\eta$ ) with the boundary conditions:

$$
\begin{array}{ll}
\Theta=1, \Phi=1, f=1, f^{\prime}=0 & \text { at } \eta=0 \\
\Theta=0, \Phi=0, f=0 & \text { at } \eta=\infty
\end{array}
$$

\section{Method of Solution}

An examination of the similarity equations (20) - (22) shows that they are highly coupled. To linearize and make them tractable, we seek the perturbation series solutions of the form

$$
\begin{aligned}
f^{*} & =f_{o}^{*}+\varepsilon f_{1}^{*}+\ldots \\
\Theta^{*} & =\Theta_{o}^{*}+\varepsilon \Theta_{1}^{*}+\ldots \\
\Phi^{*} & =\Phi_{o}^{*}+\varepsilon \Phi_{1}^{*}+\ldots
\end{aligned}
$$

where $\varepsilon=\frac{1}{f_{w}^{2}}, f_{w}=\frac{V_{w}}{(k v)^{1 / 2}}$ is the suction/injection parameter defined as the wall mass transfer coefficient. Practically, $f_{w}=f(0) . f_{w}>0$ corresponds to suction (i.e $\left.V_{w}<0\right), f_{w}<0$ corresponds to injection/blowing (i.e. $V_{w}>0$ ), and $f_{w}=0$ corresponds to an impermeable surface (i.e. $V_{w}=0$ ), as seen in Cortell[14]. 
Substituting these in equations (20)-(22) leads to more complex and intractable similarity solutions. Therefore, to have solutions that may give approximate pictures of the flow, we modified the equations (20)- (22) as:

$$
\begin{aligned}
& f^{\prime \prime \prime}+f f^{\prime \prime}-\left(f^{\prime}\right)^{2}-M_{1}^{2} f^{\prime}+\varepsilon G r(\Theta+N \Phi)=0 \\
& \frac{1}{\operatorname{Pr}} \Theta^{\prime \prime}+f \Theta '-\Theta f^{\prime}+\varepsilon \lambda \Theta=0 \\
& \frac{1}{S c} \Phi^{\prime \prime}+f \Phi^{\prime}-\Phi f^{\prime}-\varepsilon \delta_{1}^{2} \Phi=0
\end{aligned}
$$

and (26)-(28) as:

$$
\begin{aligned}
& f=1+\varepsilon f_{1}+\ldots \\
& \Theta=\Theta_{o}+\varepsilon \Theta_{1}+\ldots \\
& \Phi=\Phi_{o}+\varepsilon \Phi_{1}+\ldots
\end{aligned}
$$

Now, by equations (31) - (33), equations (28) - (30) with the boundary conditions, equations (23) and (24) give

$$
\begin{aligned}
& \frac{1}{\operatorname{Pr}} \Theta_{o}{ }^{\prime \prime}+\Theta_{o}{ }^{\prime}=0 \\
& \frac{1}{S c} \Phi_{o}{ }^{\prime \prime}+\Phi_{o}^{\prime}=0
\end{aligned}
$$

with the boundary conditions

$$
\begin{array}{ll}
\Theta_{o}=1, \Phi_{o}=1 & \text { at } \eta=0 \\
\Theta_{o}=0, \Phi_{o}=0 & \text { at } \eta=\infty
\end{array}
$$

for the zeroth order, and

$$
\begin{aligned}
& f_{1}{ }^{\prime \prime}+f_{1}{ }^{\prime \prime}-M_{1}^{2} f_{1}{ }^{\prime}=-G r\left(\Theta_{o}+N \Phi_{o}\right) \\
& \frac{1}{\operatorname{Pr}} \Theta_{1}{ }^{\prime \prime}+\Theta_{1}{ }^{\prime}=-f_{1} \Theta_{o}{ }^{\prime}+\Theta_{o} f_{1}{ }^{\prime}-\lambda \Theta_{o} \\
& \frac{1}{S c} \Phi_{1}{ }^{\prime}+\Phi_{1}{ }^{\prime}=-f_{1} \Phi_{o}{ }^{\prime}+\Phi_{o} f_{1}{ }^{\prime}+\delta \Phi_{o}
\end{aligned}
$$

with the boundary conditions

$$
\begin{array}{ll}
\Theta_{1}=0, \Phi_{1}=0, f_{1}=0, f_{1}^{\prime}=0 & \text { at } \eta=0 \\
\Theta_{1}=0, \Phi_{1}=0, f_{1}=0 & \text { at } \eta=\infty
\end{array}
$$

for the first order

Furthermore, we define the heat transferred rate (or local Nusselt number $N u$ ); mass transfer rate (or the local Sherwood number $S h$ ), and the skin friction $C_{f}$ in the dimensionless form as:

$$
\begin{aligned}
& C_{f}=\frac{\tau_{w}}{\frac{1}{2} \rho U_{\infty}^{2}}=f^{\prime \prime}(0) \\
& N u=\frac{q_{y}}{k\left(T_{w}-T_{\infty}\right)}=-\Theta^{\prime}(0) \\
& S h=\frac{J_{w} x}{D\left(C_{w}-C_{\infty}\right)}=-\Phi^{\prime}(0)
\end{aligned}
$$

where

$$
\tau_{w}=\left.\mu \frac{\partial u}{\partial y}\right|_{y=0},
$$




$$
q_{y}=-\left.k \frac{\partial T}{\partial y}\right|_{y=0}
$$

is the heat flux at the wall of the plate, and

$$
J_{w}=-\left.D \frac{\partial T}{\partial y}\right|_{y=0}
$$

mass flux at the wall of the surface

Equations (34)-(45) are solved and graphs plotted using the Mathematica 9.0 computational software.

\section{Results and Discussion}

The problem of MHD steady mixed convective flow of a chemically reacting viscous incompressible flow over a linearly stretching sheet under the influence of heat generation/absorption, thermal radiation and constant suction/injection is investigated. The effects of Hartmann number, Raleigh number, thermal differentials, chemical reaction rate, suction and Prandtl number are examined, and the results shown graphically and quantitatively in Fig.1-Fig.15, and Table 1-Table 7, respectively. For realistic constant values of $\chi^{2}=0.1, \mathrm{Sc}=0.1, \Delta=0.1$ and increasingly varied values of $\mathrm{M}^{2}=0.1,0.5,1.0,5.0,10.0 ; \mathrm{Ra}=0.1,0.3,0.5,0.7,0.9 ; \mathrm{Gr}=0.1,0.5,1.0,5.0,10.0 ; \delta_{1}{ }^{2}=0.1,0.5,1.0,5.0$, $10.0 ; \epsilon=0.01,0.03,0.05,0.07,0.09 ; \mathrm{Pr}=0.1,0.3,0.5,1.0,1.2 ; \mathrm{N}=0.1,0.5,1.0,1.5,3.0$ the analysis of results shows that $\mathrm{M}^{2}$ increases the concentration and velocity but decreases the temperature, Nusselt number and Sherwood number; Ra increases the temperature but decreases the Nusselt number; $\mathrm{Gr}$ increases the concentration, temperature and velocity but decreases the Sherwood number and skin friction; $\delta_{1}{ }^{2}$ increases the concentration but decreases the Sherwood number; $\epsilon$ decreases the concentration, velocity, Sherwood number and skin friction; Pr decreases the temperature, velocity and Sherwood number but increases the Nusselt number and skin friction; $\mathrm{N}$ decreases the concentration, temperature, Sherwood number and skin friction but increases the velocity.

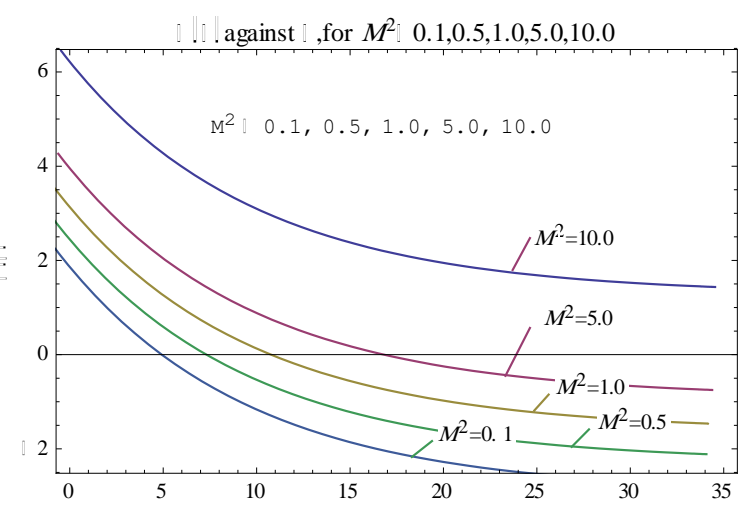

Fig.1: Concentration-Magnetic field profiles 


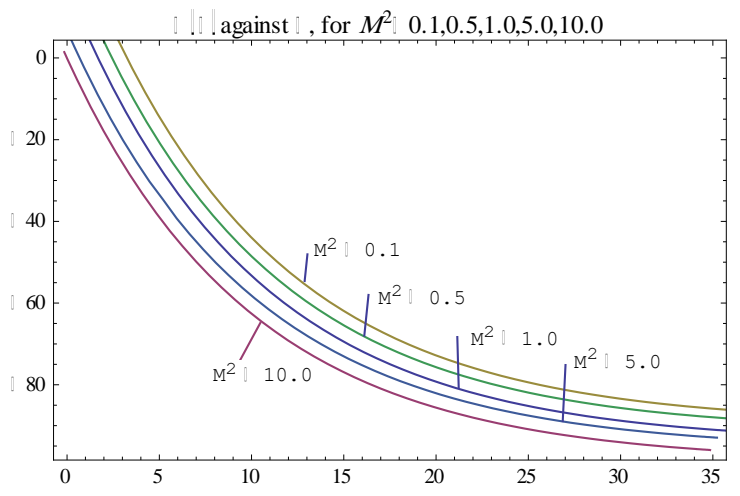

Fig.2: Temperature-Magnetic field profiles

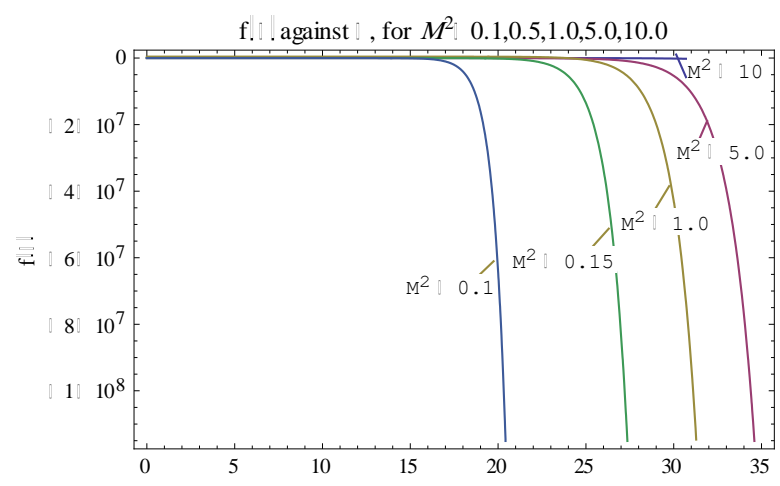

Fig.3: Velocity-Magnetic field profiles

Table 1: Nusselt Number and Sherwood Number for varied values of $\mathrm{M}^{2}$
$\mathrm{M}^{2}=0.1$
$\mathrm{M}^{2}=0.5$
$\mathrm{M}^{2}=1.0$
$\mathrm{M}^{2}=5.0$
$\mathrm{M}^{2}=10.0$
$\begin{array}{lc}\mathrm{Nu} & 0.224486179 \\ \mathrm{Sh} & 1.96537730\end{array}$
0.198690529
0.195630011
0.192694601
0.192276937
0.48159320
0.144369540
$-0.1730174332-0.216226055$

On the assumption that the fluid is electrolytic; therefore magnetically susceptible makes it exists as ions, whose motion in the magnetic field produces electric currents. The magnetic field in turn influences the electric currents to generate a mechanical force [the Lorentz] that gives the flow a new orientation. Fig. 1 and Fig. 3 show that magnetic field increases the concentration and velocity of the flow field, while Fig. 2 and Table 1 show that magnetic field decreases the temperature, Nusselt number and Sherwood number. These results agree with [15, 19, 24].

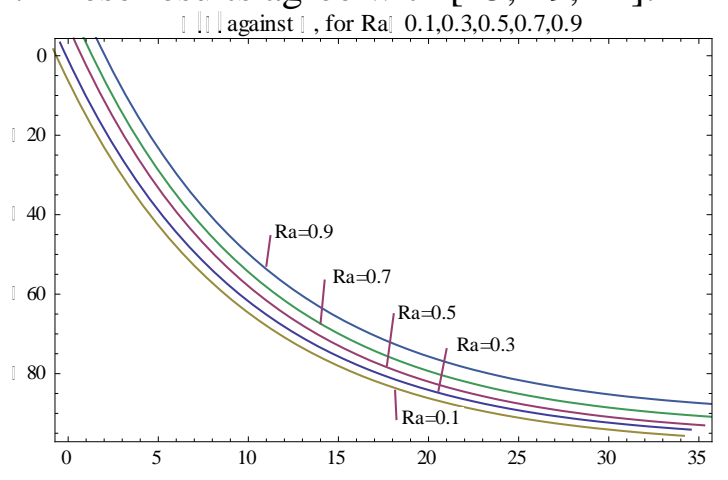

Fig.4: Temperature-Raleigh number profiles 


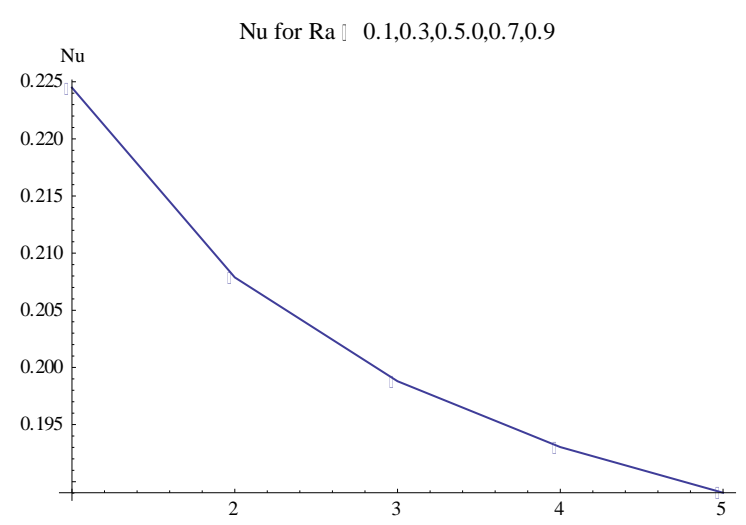

Fig.5: Nusselt number- Raleigh number profile

Furthermore, through radiation heat in the form of electromagnetic waves are emitted from the sheet into the fluid, and this leads to a convective flow. Non-dimensionally, this is expressed as the Raleigh number, which has its toll on the flow. Fig.4 and Fig.5 show the Raleigh number increases the temperature but decreases the Nusselt number of the flow field. These results are in consonance with $[15,22]$.

\begin{tabular}{ccccccc}
\multicolumn{8}{c}{ Table 2: Concentration-Grashof Number Distributions } \\
$\eta$ & $G r=0.1$ & $G r=0.5$ & $G r=1.0$ & $G r=5.0$ & $G r=10.0$ \\
0.0 & 0.062962685 & 0.210693889 & 0.611685383 & 1.87266993 & 3.71930999 \\
0.5 & 0.060440922 & 0.208172126 & 0.580627134 & 1.87014817 & 3.71678823 \\
1.0 & 0.058042147 & 0.205773352 & 0.548075099 & 1.86774940 & 3.71438945 \\
1.5 & 0.055760362 & 0.203491566 & 0.513947882 & 1.86546761 & 3.71210767 \\
2.0 & 0.053589861 & 0.201321065 & 0.478160146 & 1.86329711 & 3.70993717 \\
2.5 & 0.051525216 & 0.199256420 & 0.440622405 & 1.86123247 & 3.70787252 \\
3.0 & 0.049561265 & 0.197292469 & 0.401240794 & 1.85926851 & 3.70590857 \\
3.5 & 0.047693097 & 0.195424302 & 0.359916838 & 1.85740035 & 3.70404040 \\
4.0 & 0.045916041 & 0.193647245 & 0.316547206 & 1.85562329 & 3.70226335 \\
4.5 & 0.044225653 & 0.191956857 & 0.271023452 & 1.85393290 & 3.70057296 \\
5.0 & 0.042617706 & 0.190348910 & 0.223231742 & 1.85232496 & 3.69896501
\end{tabular}

Table 3: Temperature-Grashof Number Distributions

$\begin{array}{cccccc} & G r=0.1 & G r=0.5 & G r=1.0 & G r=5.0 & G r=10.0 \\ 0.0 & 1.001387096 & 1.009602150 & 1.019870968 & 1.102021509 & 1.204709685 \\ 0.5 & 0.199345741 & 0.916350732 & 0.926320876 & 1.008276492 & 1.110942525 \\ 1.0 & 0.825281282 & 0.826334356 & 0.834372383 & 0.916365822 & 1.019035813 \\ 1.5 & 0.732406337 & 0.736551822 & 0.746646686 & 0.828675368 & 0.931349252 \\ 2.0 & 0.647050096 & 0.652976440 & 0.663115526 & 0.745173100 & 0.847850258 \\ 2.5 & 0.566658083 & 0.573456767 & 0.583629075 & 0.665709159 & 0.768388911 \\ 3.0 & 0.490528597 & 0.497817351 & 0.508014194 & 0.590111371 & 0.692793120 \\ 3.5 & 0.418291688 & 0.425878495 & 0.436093410 & 0.518203459 & 0.620886728 \\ 4.0 & 0.349684390 & 0.357463186 & 0.367691527 & 0.449811318 & 0.552495748 \\ 4.5 & 0.284491498 & 0.292400109 & 0.302638611 & 0.384765893 & 0.487451221 \\ 5.0 & 0.222524518 & 0.230524980 & 0.240771381 & 0.322904569 & 0.425590611\end{array}$




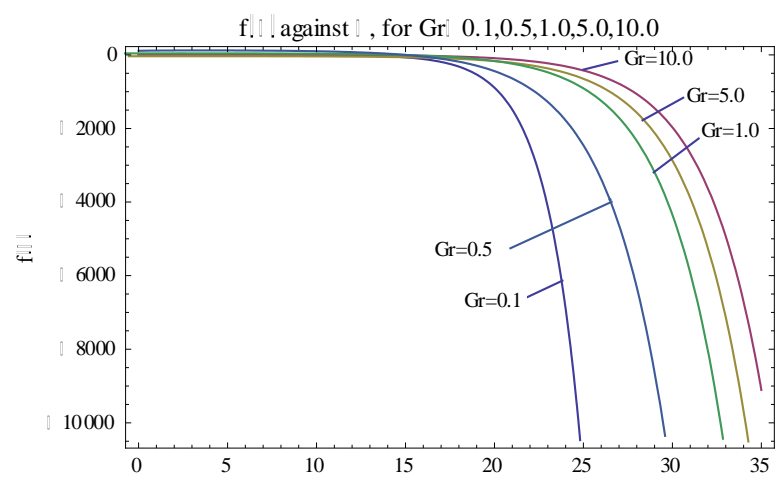

Fig.6: Velocity-Grashof Number profiles

Table 4: Sherwood Number and Skin friction for varied values of Grashof Number

$$
\begin{array}{cccccc} 
& \mathrm{Gr}=0.1 & \mathrm{Gr}=0.5 & \mathrm{Gr}=1.0 & \mathrm{Gr}=5.0 & \mathrm{Gr}=10.0 \\
\text { Sh } & 0.196537730 & -0.081471628 & -0.4289833274 & -3.209076918 & -6.684193906 \\
\text { Cf } & -0.019078550 & -0.103442519 & -0.208892003 & -1.05226878 & -2.10594202
\end{array}
$$

Usually, there is a thermal gradient between the external/environmental temperature and the fluid ambient/equilibrium temperature. This temperature gradient, in the presence of gravitational force leads to free convective force, expressed as the Grashof number. This force gives buoyancy, an energizing and a lifting force to the fluid particles. Tables 2 and Table 3, and Fig. 6 show that the Grashof number increases the concentration, temperature and velocity but decreases the Sherwood number and skin friction of the flow field. These results align with [19].

\begin{tabular}{ccccccc}
\multicolumn{6}{c}{ Table 5: Concentration-Chemical Reaction rate Distributions } \\
$\eta$ & $\delta 2=0.1$ & $\delta 2=0.5$ & $\delta 2=1$ & $\delta 2=5.0$ & $\delta 2=10$ \\
0.0 & 0.06296268532 & 0.1270822222 & 0.6116853838 & 0.8484270123 & 1.649921223 \\
0.5 & 0.06044092256 & 0.1183485893 & 0.5806271342 & 0.7698098404 & 1.493655675 \\
1.0 & 0.05804214762 & 0.1100409007 & 0.5480750993 & 0.6950268733 & 1.345011287 \\
1.5 & 0.05576036232 & 0.1021383829 & 0.5139478822 & 0.6238911144 & 1.203616371 \\
2.0 & 0.05358986100 & 0.0946212754 & 0.4781601469 & 0.5562246875 & 1.069117367 \\
2.5 & 0.05152521627 & 0.0874707815 & 0.4406224056 & 0.4918583912 & 0.941177957 \\
3.0 & 0.04956126546 & 0.0806690214 & 0.4012407942 & 0.4306312762 & 0.819478225 \\
3.5 & 0.04769309766 & 0.0741989870 & 0.3599168384 & 0.3723902428 & 0.703713860 \\
4.0 & 0.04591604148 & 0.0680444999 & 0.3165472066 & 0.3169896581 & 0.593595389 \\
4.5 & 0.04422565335 & 0.0621901707 & 0.2710234521 & 0.2642909919 & 0.488847459 \\
5.0 & 0.04261770642 & 0.0566213605 & 0.2232317420 & 0.2141624699 & 0.389208146
\end{tabular}

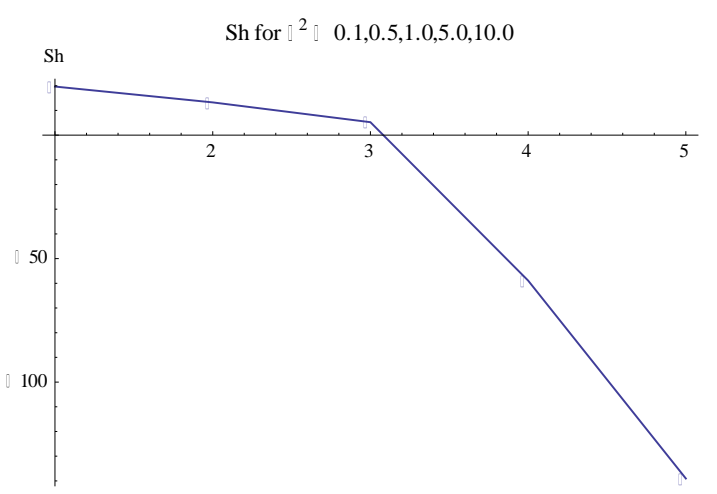

Fig.7: Sherwood Number-Chemical Reaction rate profile

Even so, a chemical reaction leads to the depletion of the chemicals in a fluid. The chemical reaction may be exothermic or endothermic, and whichever occurs, the heat content of the system is either increased or decreased. Table 5 and Fig.7 show that the chemical reaction rate increases the concentration but decreases the Sherwood number of the flow field. Also, as seen in Fig.7, the drop in the Sherwood number is gradual for $\delta_{1}^{2} \leq 1.0$ and drastic for $\delta_{1}^{2} \geq 5$. 


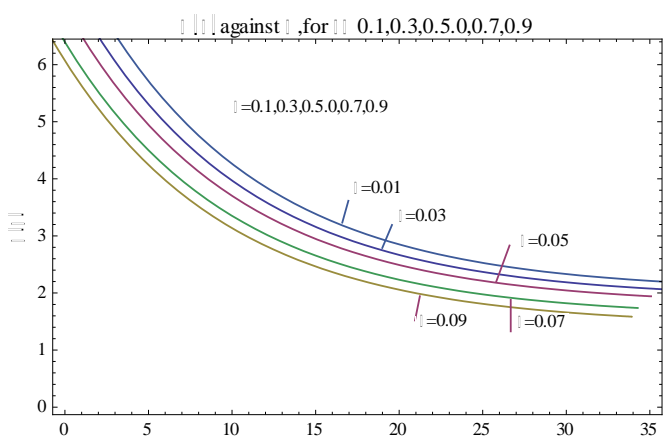

Fig.8: Concentration-Suction parameter profiles

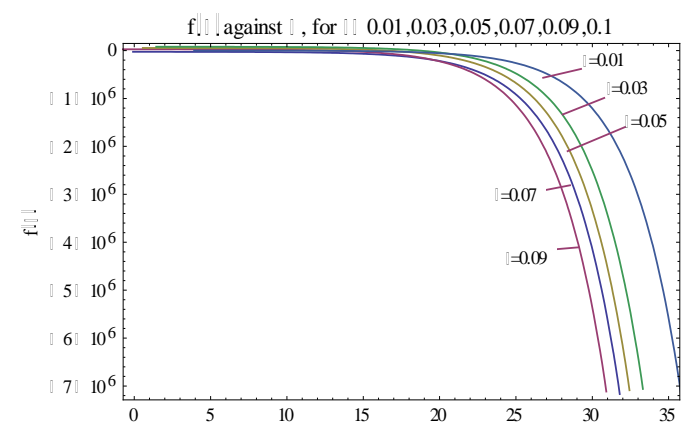

Fig.9: Velocity-Suction parameter profiles

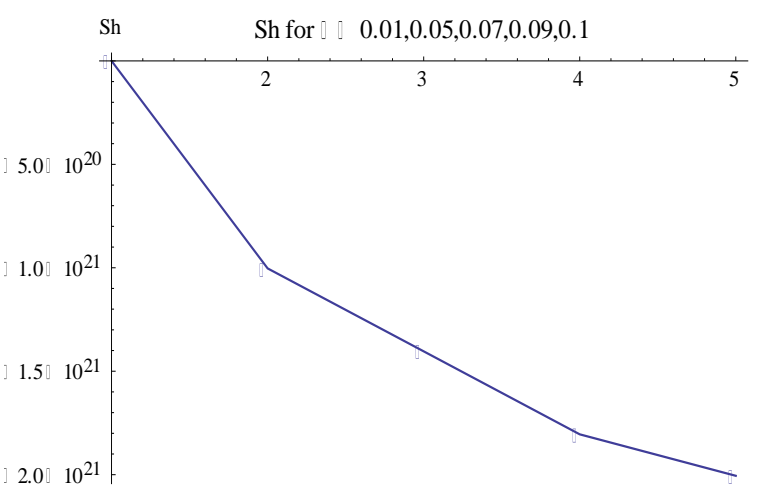

Fig.10: Sherwood Number-Suction parameter profiles

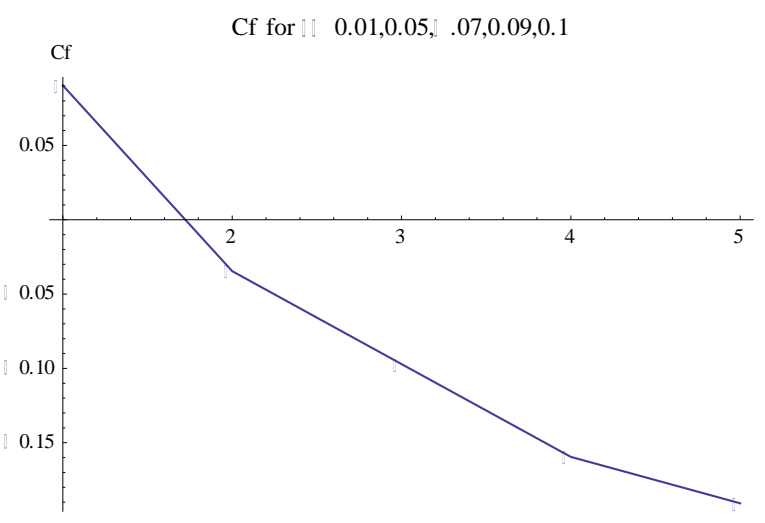

Fig.11: Skin Friction-Suction parameter profile

Moreover, the calculation shows that $f(0)=f_{w}=1.91876$, and $\epsilon=0.27$. These depict that this problem is suction induced. Upon this, the fluid moves towards the stretching sheet. And, this has its attendant effects on the flow field. Fig.8 - Fig.11 show that the suction parameter decreases the concentration, velocity, Sherwood number and skin friction, respectively. 


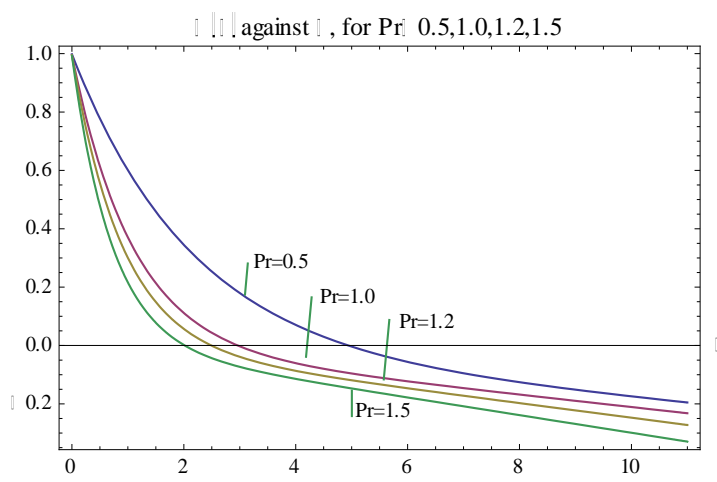

Fig.12: Temperature-Prandtl Number profiles

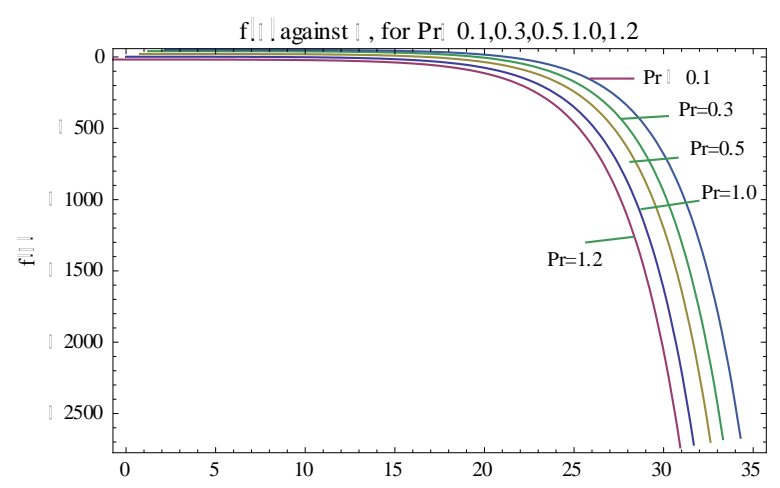

Fig.13: Velocity-Prandtl Number profiles

Table 6: Nusselt number, Sherwood number and Skin friction for varied values of Prandtl Number

$\begin{array}{cccccc} & \operatorname{Pr}=0.1 & \operatorname{Pr}=0.3 & \operatorname{Pr}=0.5 & \operatorname{Pr}=1.0 & \operatorname{Pr}=1.2 \\ \text { Nu } & 0.224486179 & 0.335408463 & 0.4939306726 & 0.948725418 & 1.135620887 \\ \text { Sh } & 1.965377303 & 0.000307753 & -0.2512329225 & -0.321365862 & -0.32296445 \\ \text { Cf } & -0.190785506 & 0.061703694 & 0.148617766 & 2.323384015 & \end{array}$

Additionally, Prandtl number is a dimensionless representation of the ratio of kinematic/momentum diffusivity to thermal diffusivity. It increases when the momentum diffusivity dominates the thermal diffusivity. Fig.12 and Fig.13, and Table 6 depict that the temperature, velocity and Sherwood number decrease as the Prandtl number increases, while the Nusselt number and skin friction increase as the Prandtl number increases. These results are in agreement with $[12,15]$.

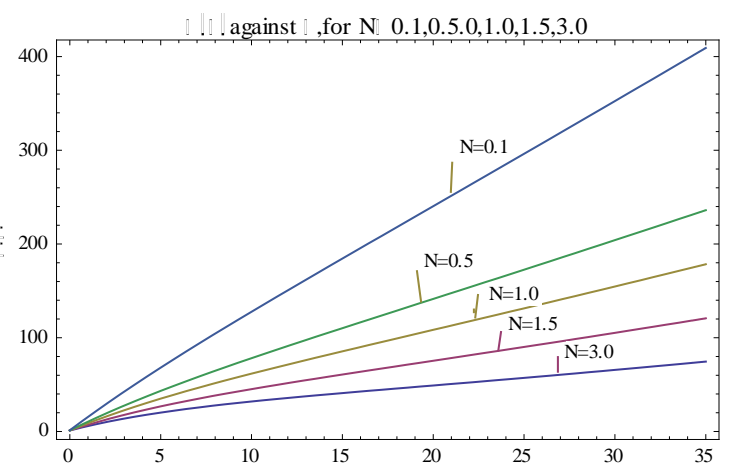

Fig.14: Concentration-Buoyancy ratio parameter profiles 


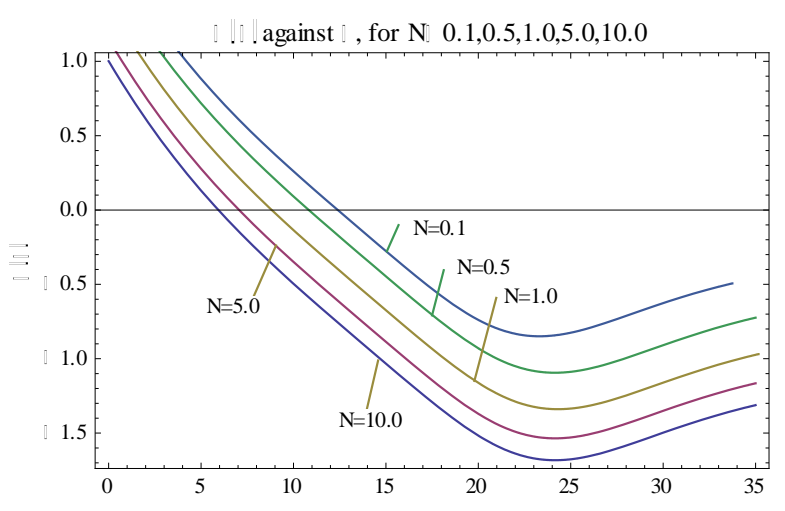

Fig. 15: Temperature-Buoyancy ratio parameter profiles

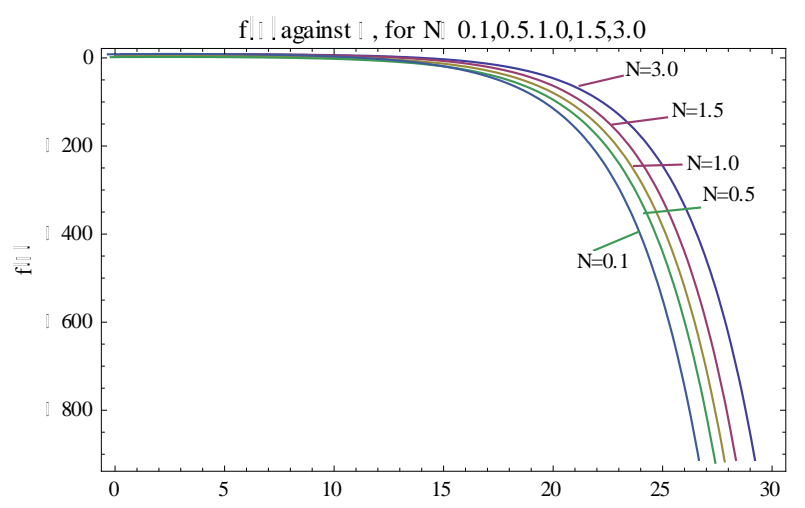

Fig.16: Velocity-Buoyancy ratio parameter profiles

Table 7: Sherwood Number and Skin friction for varied values of Buoyancy ratio parameter
$\mathrm{N}=0.1$
$\mathrm{N}=0.5$
$\mathrm{N}=1.0$
$\mathrm{N}=1.5$
$\mathrm{N}=3.0$
Sh 1.965377303
1.712641522
1.396721796
1.080802070
0.133042891
Cf -0.190785506
$-0.280546680$
$-0.392748147-0.504949614$
$-0.841554019$

Similarly, buoyancy ratio depends on the strength of the concentration differential and thermal differential compared. It rises when the concentration gradient increases. Fig.14, Fig. 15 and Table 7 show that concentration, temperature, Sherwood number and skin friction decrease as the buoyancy ratio parameter increases while the velocity increases as the buoyancy ratio increases. These results are in alignment with [23].

\section{Conclusion}

Thermally driven MHD steady mixed convective chemically reacting flow of a viscous incompressible fluid over a linearly stretching sheet with the attendant heat generation/absorption, suction/injection and thermal radiation is examined. The overall analysis shows that the increase in the

- magnetic field parameter increases the concentration and velocity but decreases the temperature, Nusselt number and Sherwood number;

- the Raleigh number increases the temperature but decreases the Nusselt number;

- Grashof number increases the concentration, temperature and velocity but decreases the Sherwood number and skin friction;

- chemical reaction rate increases the concentration but decreases the Sherwood number;

- $\quad$ suction decreases the concentration, velocity, Sherwood number, and skin friction;

- Prandtl number decreases the temperature, velocity, Sherwood number but increases Nusselt number and skin friction;

- buoyancy ratio parameter decreases the concentration, temperature, Sherwood number and skin friction but increases the velocity. 


\section{References}

[1] S.S. Motsa and P. Sibanda, On the solution of nonlinear stretching sheet by an efficient semianalytic technique, International Journal of Numerical Methods in Fluids 68 (2012), pp. 1524-1537. DOI: $10.1002 /$ fld.2541

[2] M. Ferdows, Md Khan Shakhaoath, Md Alam Mahmud,Shuyu Sun, MHD mixed convective boundary layer flow of a nano-fluid through a porous medium due to an exponentially stretching sheet. Mathematical Problems in Engineering (2012), pp. 1-21. DOI: 10.1155/2012/408528

[3] M. Wahiduzzana, Md Khan Shakhaoath, P. Biswas, Ifsans Karim, M.S. Uddin, Viscous dissipation and radiation effects on MHD boundary layer flow of a nano-fluid past a rotating stretching sheet. Journal Applied Mathematics 6 (2015), pp. 547-567.

[4] S. Nadeem, S.T. Hussain, L. Changhoon, Flow of a Williamson fluid over a stretching sheet. Brazilian Journal of Chemical Engineering 30(3) (2013). DOI: http://dx.doi.org/10.1590/S010466322013000300019

[5] S. Nadeem, U.I.R. Haq, Noreen Sher Akhbe, MHD 3-D flow of Casson Nano-fluid past a linearly stretching sheet with convective boundary conditions. IEEE Transact. on Nano-technology 3(1) (2014), pp. 109-115. DOI: 10.1109/tnano.2013.223735

[6] F.G. Awad, S.M.S. Ahmed, P. Sibanda, M. Khumal, The effects of thermo-phoresis on unsteady Oldroyd-B Nano-fluid flow over a stretching surface PLOS ONE. (2015). DOI: 10.1371/journal.pone.0135914

[7] B.C. Sakiadis, Boundary layer behaviour on continuous solid surface. American Institute of Chemical Engineering Journal 7(2) (1961), pp. 221-225

[8] E.M.A. Elbashbeshy, M.A.A. Bazid, Heat transfer on a continuously moving plate embedded in non-Darcian porous medium. International Journal of Heat and Mass Transfer 43(2000), pp. 3087 3092.

[9] P.D. Ariel, T. Hayat, S. Asghar, The flow of an elasto-viscous fluid past a stretching sheet with partial slip. Acta Mechanica 187 ( 2006), pp. 1220-1228

[10] R. Kandasamy, T. Hayat, S. Obaidat, Group theory transformation for Soret and Dufour effects on free convective heat and mass transfer with thermo-phoresis and chemical reaction over a porous stretching surface in the presence of heat source/sink. Nuclear Engineering and Design 241(6) (2011), pp. 2155-2161.

[11] H. Xu, An explicit analytic solution for convective heat transfer in an electrically conducting fluid at a stretching surface with uniform free stream. International Journal of Engineering Science 43 (2005), pp. 859-874.

[12] R. Cortell, Flow and heat transfer of a fluid through a porous medium over a stretching surface with internal heat generation/absorption and suction/blowing. Fluid Dynamics Research 37 (2005), pp. 231-245.

[13] M.A. Seddeek, A.A. Almushigeh, Effects of radiation and variable viscosity on MHD free convective flow and mass transfer over a stretching sheet with chemical reaction. Applied Mathematics and Computation 5(1) (2010), pp. 181-197.

[14] J.I. Oahimire, B.I. Olajuwon, Hydrodynamic flow near a stretching sheet with variable thermal conductivity and heat source/sink. Journal of Applied Science and Engineering 11(3) (2013), pp. 331-341.

[15] S. Shateyi, A numerical approximation to MHD flow of Maxwell fluid past a vertically stretching sheet in the presence of thermo-phoresis and chemical reaction. Boundary Value Problems 2013 (2013), pp. 196

[16] P. Vyas, N. Srivastava, Radiative MHD flow over a non-isothermal stretching sheet in a porous medium. Applied Mathematical Science 4(49.52) (2009), pp. 2475-2484.

[17] S.S. Motsa, S. Shateyi, Successive linearization analysis of unsteady heat and mass transfer from a stretching surface embedded in a porous medium with suction/injection and thermal radiation effects. Canadian Journal of Chemical Engineering 9999 (2011), pp. 1-13.

[18] P. Vyas, N. Srivastava, On dissipative radiative MHD boundary layer flow in a porous medium over a non-isothermal stretching sheet. Journal of Applied Fluid Mechanics 5(4) (2012), pp. 23-31. 
[19] S. Ibrahim Mohammed, Effects of chemical reaction on dissipative radiative MHD flow through a porous medium over a non-isothermal stretching sheet. Journal of Industrial Mathematics 2014 (2014) DOI: http://dx.doi.org/10.1155/2014/243148

[20] T. Hayat, M.B. Ashraf, S.A. Shehzad, A. Alsaedi, Mixed convective flow of Casson Nano-fluid over a stretching sheet with convectively heated chemical reaction and heat source/sink. Journal of Applied Fluid Mechanics 8(4) (2015), pp. 803-813.

[21] N. Sandeep, C. Sulochana, C.S.K. Raju, M.J. Babu, Sugunamma V., Unsteady boundary layer flow of thermo-phoretic MHD Nano-fluid past a stretching sheet with space and time dependent internal heat source/sink. Application and Applied Mathematics. (AAM) 10(1) (2015), pp. 312-327. [22] G.R. Sreedevi, R. Raghavendra Rao, D.R.V. Prasada Rao, A.J. Chamkha, Combined influence of radiation absorption and hall current effects on MHD double-diffusive free convective flow past a stretching sheet. Ains Shams Engineering Journal (2016), pp. 383-397.

[23] Adetunji Adeniyan, Soret-Dafour and stress work effects on hydro-magnetic free convection of a chemically reactive stagnation-slip flow and heat transfer towards a stretching vertical surface with heat generation and variable thermal conductivity. Asian Journal of Mathematics and Applications (2016), pp. 1-23.

[24] H.S. Takhar, A.J. Chamkha, G. Nath, Flow and mass transfer on a stretching sheet with a magnetic field and chemically reactive species. International. Journal of Engineering Science. 38 (2000), pp. 1304-1314.

[25] O.D. Makinde, P. Sibanda, Effects of chemical reaction on boundary layer flow past a vertically stretching surface in the presence of internal heat generation. International Journal of Numerical Methods for Heat and Fluid Flow. 12(6) (2011), pp. 779-792.

[26] W.G. Vincentti, G.H. Krugger, Introduction to Physical Gas-dynamics, New York, (1969).

[27] R. Siegel, J.R. Howel, Thermal Radiation Heat Transfer, Hemisphere, New York, (1972).

[28] K.D. Alagoa, G. Tay, T.M. Abbey, Radiative and free convective effects of a MHD flow through a porous medium between infinite parallel plates with time-dependent suction. Astrophysical and Space Science 260 (1998), pp. 455-468.

[29] C. Israel-Cookey, C. Nwaigwe, Unsteady MHD of a radiating fluid over a moving heated porous plate with time-dependent suction. American Journal Scientific and Industrial Reearch. 1(1) (2010), pp. 85-95. 